\title{
Bioengineering a light-responsive encapsulin nanoreactor: a potential tool for photodynamic therapy
}

\author{
Dennis Diaz ${ }^{1}$, Xavier Vidal ${ }^{2,3}$, Anwar Sunna $^{1,4}$ \& Andrew Care ${ }^{1,4 *}$
}

1Department of Molecular Sciences, Macquarie University, NSW 2109, Australia

2Department of Physics and Astronomy, Macquarie University, NSW 2109, Australia ${ }^{3}$ Fraunhofer Institut für Angewandte Festkörperphysik (IAF), Tullastrasse 72, 79108 Freiburg, Germany

${ }^{4}$ Australian Research Council Centre of Excellence for Nanoscale BioPhotonics, Macquarie University, NSW 2109, Australia.

* Corresponding author: Andrew Care

Department of Molecular Sciences

Macquarie University

Sydney, NSW 2109

Australia

Phone:

+61298506267

Fax:

+61298508313

Email:

andrew.care@mq.edu.au 


\section{Abstract}

Encapsulins, a prokaryotic class of self-assembling protein nanocompartments, are being re-engineered to serve as 'nanoreactors' for the augmentation or creation of key biochemical reactions. However, approaches that allow encapsulin nanoreactors to be functionally activated with spatial and temporal precision is lacking. We report the construction of a light-responsive encapsulin nanoreactor for "on-demand" production of reactive oxygen species (ROS). Herein, encapsulins were loaded with the fluorescent flavoprotein mini-Singlet Oxygen Generator (miniSOG), a biological photosensitizer that is activated by blue-light to generate ROS, primarily singlet oxygen $\left({ }^{1} \mathrm{O}_{2}\right)$. We established that the nanocompartments stably encased miniSOG, and in response to blue-light were able to mediate the photoconversion of molecular oxygen into ROS. Using an in vitro model of lung cancer, ROS generated by the nanoreactor was shown to trigger photosensitized oxidation reactions that exerted a toxic effect on tumour cells, suggesting utility in photodynamic therapy. This encapsulin nanoreactor thus represents a platform for the light-controlled initiation and/or modulation of ROSdriven processes in biomedicine and biotechnology.

\section{Keywords}

encapsulin, self-assembly, compartmentalisation, nanoreactor, photosensitizer, reactive oxygen species (ROS), photodynamic therapy, protein delivery 


\section{Introduction}

In the era of synthetic biology, there is increasing interest in re-engineering sub-cellular compartments (e.g. organelles) into designer 'nanoreactors' that can augment/enhance existing biological reactions, or enable the creation of entirely new synthetic reactions. ${ }^{1-3}$ This includes protein compartments that encase enzymes within a selectively permeable protein shell that self-assembles from multiple protein subunits. 4, 5 This design isolates and promotes specialized reactions by placing catalytic proteins, their substrates and cofactors within close proximity of each other; regulating the influx and efflux of molecules; preventing the escape of volatile or toxic reaction intermediates; and creating distinct microenvironments that can protect, stabilise and improve the function of cargo proteins. ${ }^{6}$

Encapsulins are a newly established class of prokaryotic protein nanocompartments, which self-assemble from identical protein subunits into semi-permeable icosahedral protein shells with $T=1$ (60 subunits, $20-24 \mathrm{~nm}), T=3$ (180 subunits, $30-32 \mathrm{~nm}$ ) or $T$ $=4$ (240 subunits, $43 \mathrm{~nm}$ ) symmetries. ${ }^{3,7-9}$ In nature, they encase functional cargo proteins that help their microbial hosts maintain iron homeostasis, cope with oxidative and nitrosative stress, and/or safely derive energy from ammonium. ${ }^{10-12}$ Moreover, encapsulins recognise and selectively encapsulate native cargo proteins that display a short encapsulation signal peptide (ESig), a distinct mechanism that can be adapted to instead package foreign cargo, reprogramming the nanocompartments' functionality. $7,12,13$

The structural modularity, functional programmability and widespread prevalence of encapsulins in prokaryotes, makes them an ideal toolbox for nanoreactor construction. By packaging non-native enzymes, encapsulins have been recently reprogrammed to stabilize the synthesis of precursors used in the production of pharmaceutical opioids; and to safely compartmentalize the orthogonal synthesis of toxic melanin thereby mimicking melanosome organelles found in mammals. ${ }^{1,2}$ However, the capacity to selectively control the activity of such encapsulin nanoreactors is lacking. Herein, we explore the construction of an encapsulin nanoreactor that generates reactive oxygen species (ROS) in response to external light stimulation, permitting precise temporal and spatial control over its activity. 
As a first step towards designing a light-responsive encapsulin nanoreactor, we elected to load nanocompartments with mini-Singlet Oxygen Generator (miniSOG). miniSOG is a flavin mononucleotide (FMN)-based fluorescent protein derived from the light-oxygen-voltage-sensing domain of Arabidopsis thaliana phototropin-2. Via its FMN chromophore, miniSOG can be activated by blue-light to photoconvert molecular oxygen $\left({ }^{3} \mathrm{O}_{2}\right)$ into ROS, primarily singlet oxygen $\left({ }^{1} \mathrm{O}_{2}\right) .{ }^{14}$ Consequently, in a process called 'photosensitization', ${ }^{1} \mathrm{O}_{2}$ generated by light-induced miniSOG has been exploited in various applications. These include the photooxidation of contrast agents in bioimaging, photoredox activation of prodrugs, photomodulation of ROS-activated signalling pathways, chromophore-assisted light inactivation of proteins in optogenetics, and photoinduced destruction of cancer cells in photodynamic therapy (PDT). 15-22

In this communication, we present the confinement of ESig-tagged miniSOG inside Thermotoga maritima encapsulin, reprogramming it to serve as a light-responsive nanoreactor for "on demand" ROS generation. We show the capacity of this first-of-itskind encapsulin nanoreactor to house miniSOG and its blue-light induced photoconversion of ${ }^{3} \mathrm{O}_{2}$ to ${ }^{1} \mathrm{O}_{2}$. We further characterize the effect encapsulation has on miniSOG's ${ }^{1} \mathrm{O}_{2}$-generating function, and the impact of prolonged ROS generation on the nanocompartments' structure and stability. As a proof-of-concept, the ROS produced by the light-activated nanoreactor is demonstrated to trigger photosensitized oxidation reactions that exert a phototoxic effect on lung cancer cells, indicating potential utility in PDT. 


\section{Materials and methods}

\section{Materials}

All chemicals and reagents used in this study were purchased from Sigma-Aldrich unless stated otherwise.

\section{Molecular biology and cloning}

All inserts were codon optimized for expression in Escherichia coli and custom synthesized as gBlock Gene Fragments (Integrated DNA Technologies). A surfaceexposed loop region between residues 138 and 139 of the encapsulin from Thermotoga maritima (Tm) (Uniprot: Q9WZP3) was modified with a hexahistidine tag (GGGGGGHHHHHHGGGGGG) (his-tag) as previously described. 23 For the encapsulation of miniSOG inside his-tagged encapsulin (Enc), the protein was Cterminally tagged with a minimized $T m$ encapsulation signal (ESig, GGSENTGGDLGIRKL), ${ }^{24}$ resulting in ESig-tagged miniSOG (mSOG). ${ }^{25}$ To generate expression vectors, Enc was ligated into pETDuet-1 (Merck, USA) via Ncol/BamHI restrictions sites, while $m S O G$ was inserted into pACYC-Duet-1 (Merck, USA) via $\mathrm{Ndel} / \mathrm{Bg}$ Ill restriction sites. Escherichia coli $\alpha$-Select (Bioline, UK) was used as a host for plasmid propagation. Gene insertion was verified by PCR using the primer pairs pETUpstream/DuetDOWN or DuetUP2/T7 Terminator (Merck) with Enc or mSOGcontaining plasmids as template DNA. All plasmids constructed for this study are summarized in Supplementary Table S1. Escherichia coli BL21 (DE3) cells (New England Biolabs, USA) were used for recombinant protein expression. For the coexpression of Enc and its intended mSOG cargo protein, cells were co-transformed with the appropriate expression plasmids, and the resulting transformants were selected on Luria-Bertani (LB) agar supplemented with carbenicillin (100 $\mu \mathrm{g} / \mathrm{ml})$ and chloramphenicol (50 $\mathrm{\mu g} / \mathrm{ml})$ (see Supplementary Table S2).

\section{Protein expression and purification}

Protein expression (or co-expression) experiments were carried out in LB medium supplemented with carbenicillin $(100 \mu \mathrm{g} / \mathrm{ml})$, chloramphenicol $(50 \mu \mathrm{g} / \mathrm{ml})$, or both. Briefly, $500 \mathrm{ml}$ LB was inoculated 1:100 with an overnight culture of cells harbouring the expression plasmid(s) of interest. Then, the culture was grown aerobically at $37^{\circ} \mathrm{C}$ to an optical density at $600 \mathrm{~nm}\left(\mathrm{OD}_{600}\right)$ of $0.5-0.8$ and induced by the addition of isopropyl-ß-D-thiogalactopyranoside (IPTG). The optimized conditions for the 
recombinant expression of all proteins in this study are outlined in Table S2. Finally, cells were harvested by centrifugation $\left(10,000 \times g 15 \mathrm{~min}, 4^{\circ} \mathrm{C}\right)$ and stored at $-30{ }^{\circ} \mathrm{C}$. For the purification of Enc and mSOG-loaded Enc (Enc-mSOG), the pellet from a 500 $\mathrm{mL}$ culture was resuspended in $50 \mathrm{~mL}$ lysis buffer $\left(20 \mathrm{mM} \mathrm{NaH}_{2} \mathrm{PO}_{4}, 300 \mathrm{mM} \mathrm{NaCl}\right.$, $40 \mathrm{mM}$ imidazole, $1 \mathrm{U} / \mathrm{mL}$ Benzonase nuclease, $\mathrm{pH}$ 7.4). Cells were lysed with a French pressure cell press and subsequently centrifuged at $10,000 \times g$ for $15 \mathrm{~min}$. The lysate was then subjected to nickel-immobilized metal affinity chromatography (IMAC) using a HisPrep ${ }^{\mathrm{TM}}$ Fast Flow 16/10 column (GE Healthcare, USA) equilibrated with equilibration buffer (20 mM NaH $\mathrm{PO}_{4}, 300 \mathrm{mM} \mathrm{NaCl}, 40 \mathrm{mM}$ imidazole, $\mathrm{pH}$ 7.4). The imidazole concentration within the equilibration buffer was increased to $260 \mathrm{mM}$ or 400 $\mathrm{mM}$ to elute Enc-mSOG and Enc, respectively. Next, eluted protein fractions were concentrated using Amicon® Ultra-15 centrifugal filter units (Merck, USA) with a 100 KDa cut-off, followed by dilution in $7 \mathrm{~mL}$ of 50 mM HEPES buffer $\mathrm{pH} 7.4$ (Chem-Supply Pty, Australia). A second purification step by size exclusion chromatography (SEC) was subsequently performed using a HiPrep ${ }^{\text {TM }}$ 26/60 Sephacryl囚 S-500 HR column (GE Healthcare, USA) and 100 mM HEPES Buffer. All purifications were carried out on an Äkta ${ }^{\mathrm{TM}}$ start or Äkta ${ }^{\mathrm{TM}}$ pure chromatography system (GE Healthcare, USA).

For the purification of free $\mathrm{mSOG}$ the unbound fraction obtained during the IMAC purification of Enc-mSOG was used. Herein, a saturated solution of ammonium sulphate was added to a final concentration of $30 \%(\mathrm{v} / \mathrm{v})$, incubated on ice for $30 \mathrm{~min}$ and spun down at $10,000 \times g$ for $15 \mathrm{~min}$. Next, ammonium sulphate was added to the supernatant to a final concentration of $50 \%(\mathrm{v} / \mathrm{v})$, inducing protein precipitation. The precipitated protein was resuspended in 100 mM HEPES buffer $(\mathrm{pH} 7.4)$ and subjected to SEC using a HiPrep ${ }^{\mathrm{TM}} 16 / 60$ Sephacryl ${ }^{\circledR}$ S-400 column (GE Healthcare, USA) using 100 mM HEPES Buffer. The fractions containing free mSOG were pooled and concentrated using Amicon Ultra-15 centrifugal filter units with a $10 \mathrm{KDa}$ cut-off. The final protein concentrations were determined by measuring absorbance at $280 \mathrm{~nm}$. Examples of purification chromatograms are provided in Supplementary Figure 1.

\section{Polyacrylamide gel electrophoresis (PAGE)}

The Bio-Rad mini-protean system (Bio-Rad laboratories, USA) was used for all SDSPAGE and Native-PAGE analysis. For SDS-PAGE, samples were diluted in $2 X$ Laemmli sample buffer with $50 \mathrm{mM}$ 1,4-dithiothreitol, heated at $95^{\circ} \mathrm{C}$ for $5 \mathrm{~min}$, loaded into pre-cast Bio-Rad Mini-PROTEAN® ${ }^{\circ} \mathrm{TX}^{\mathrm{TM}}$ gels (4-15\%) and run at $200 \mathrm{~V}$ for 30 min. For Native-PAGE, samples were diluted in 4X native sample buffer (200 mM Tris- 
$\mathrm{HCl} \mathrm{pH} \mathrm{6.8,} \mathrm{40 \%} \mathrm{glycerol,} \mathrm{and} \mathrm{0.08 \%} \mathrm{bromophenol} \mathrm{blue),} \mathrm{loaded} \mathrm{into} \mathrm{pre-cast} \mathrm{Bio-Rad}$ Mini-PROTEAN $®$ TGX ${ }^{\mathrm{TM}}$ gels (4-20\%) and run at $200 \mathrm{~V}$ for a minimum of $2 \mathrm{~h}$. In-gel fluorescence of proteins was observed with a gel documentation imager (Bio-Rad laboratories, USA). All gels were stained following the Coomassie G-250 safe stain protocol ${ }^{26}$. The densitometric intensity of protein bands in SDS-PAGE gels were quantified using ImageJ software (National Institutes of Health, USA) ${ }^{27}$.

\section{Transmission electron microscopy (TEM)}

$10 \mu \mathrm{L}$ of Enc or Enc-mSOG $(\sim 100 \mu \mathrm{g} / \mathrm{ml})$ was adsorbed onto formvar-carbon coated copper grids for 2 min and negatively stained with uranyl acetate replacement stain (UAR-EMS) for $1 \mathrm{~h}$. Grids were then washed with ultrapure water and allowed to dry for 15 min. Finally, the grids were observed under a CM10 TEM (Philips, Netherlands) operated at $100 \mathrm{kV}$ accelerating voltage.

\section{Dynamic light scattering (DLS)}

DLS data was collected on a Nano ZS90 Zetasizer (Malvern, UK). Measurements were performed at room temperature using standard cuvettes containing $1 \mathrm{~mL}$ of Enc or Enc-mSOG were diluted in 100 mM HEPES ( $\mathrm{pH} \mathrm{7.4)} \mathrm{to} \mathrm{a} \mathrm{final} \mathrm{concentration} \mathrm{0.2-0.4}$ $\mathrm{mg} / \mathrm{mL}$. The signal was averaged over 13 readings, each lasting $30 \mathrm{~s}$.

\section{Absorbance and fluorescence spectrometry}

The fluorescence excitation and emission spectra of free and encapsulated mSOG were obtained on a Cary Eclipse Fluorescence Spectrophotometer (Agilent Technologies, USA) or Fluorolog ${ }^{\circledR}$ (Horiba, Japan) using quartz cuvettes. Absorbance for protein concentration measurements was acquired at $280 \mathrm{~nm}$ on a SPECTROstar $®$ Nano Plate Reader (BMG Labtech, Germany) using UV-transparent 96 well plates.

\section{Singlet oxygen detection}

Singlet oxygen generation from free mSOG, Enc-mSOG, and unloaded Enc was detected in solution with the fluorescent probe Singlet Oxygen Sensor Green (SOSG) according to the manufacturer's protocol (Invitrogen, USA). The reaction mixture contained $500 \mathrm{nM}$ of free mSOG or encapsulated mSOG (Enc-mSOG) or 4.8 of $\mu \mathrm{M}$ unloaded Enc (in 100 mM HEPES buffer, pH 7.5), 1 MM SOSG, and 50\% deuterium oxide $\left(\mathrm{D}_{2} \mathrm{O}\right)$. The concentration of unloaded Enc is equivalent to the concentration of the nanocompartment shell present in Enc-mSOG reactions. Reaction mixtures were 
irradiated with a Chameleon-Ultra II laser (Coherent) passing through a harmonic converter to a set wavelength of $450 \mathrm{~nm}$ with an average power density of $55 \mathrm{~mW} / \mathrm{cm}^{2}$ for $10 \mathrm{~min}$. For further characterization, other irradiation times were evaluated $(0,10$, 15 and $20 \mathrm{~min}$ ). Fluorescence signals from the oxidized SOSG (excitation/emission = 485/520 nm) were measured on a PHERAstart FS (BMG Labtech, Germany) microplate reader.

\section{In vitro cytotoxicity and phototoxicity}

For in vitro cytotoxicity and phototoxicity studies, $5.0 \times 10^{3}$ A549 cells per well were seeded into 96 -well microplates and cultured at $37^{\circ} \mathrm{C}$ for $24 \mathrm{~h}$. First, the effect of laser irradiation on cell viability was investigated by exposing cells to a $450 \mathrm{~nm}$ blue laser at a power density of $55 \mathrm{~mW} / \mathrm{cm}^{2}$ for different time periods $(0,5,10$ and $15 \mathrm{~min})$. To evaluate the cytotoxicity of all protein constructions in the absence of light activation, cells were incubated with $4.8 \mu \mathrm{M}$ unloaded Enc and $500 \mathrm{nM}$ mSOG or Enc-mSOG at $37^{\circ} \mathrm{C}$ in the dark for $2,4,8$ and $12 \mathrm{~h}$. After being subjected to each of these treatments, cells were washed once with PBS to remove non-internalized protein, and fresh growth medium was added. Cells were cultivated for a further $48 \mathrm{~h}$, and cell viability was then determined using the 3-(4,5-dimethylthiazol-2-yl)-2,5-diphenyltetrazolium bromide (MTT) cell viability assay (Invitrogen, USA) according to the manufacturer's protocol 28. In phototoxicity studies, the same protocol described for cytotoxicity was performed with minor changes. Briefly, after A549 cells were treated with free mSOG, Enc-mSOG, and unloaded Enc for different times (2, 4, 8 and $12 \mathrm{~h}$ ) the medium was replaced with PBS and cells were irradiated with a $450 \mathrm{~nm}$ blue laser at $55 \mathrm{~mW} / \mathrm{cm}^{2}$ for $10 \mathrm{~min}$. Next, fresh medium was added to the cells, followed by cultivation for another $48 \mathrm{~h}$ in the dark. Cell viability was subsequently measured by MTT assay. For each experiment at least three technical replicates were performed. 


\section{Results and discussion}

Reprogramming an encapsulin nanocompartment into a light-activatable nanoreactor

In nature, Thermotoga maritima $(\mathrm{Tm})$ encapsulin packages a ferritin-like protein that facilitates the sequestration, oxidization and biomineralization of iron, indicating a physiological role in iron homeostasis and oxidative stress responses. We aimed to bioengineer a light-activatable nanoreactor for the production of ROS by reprogramming the $\mathrm{Tm}$ encapsulin to encase the photosensitizing protein miniSOG.

To selectively target miniSOG's encapsulation inside $\mathrm{Tm}$ encapsulin, its C-terminus was tagged with a functional minimized 15-amino acid Tm ESig. 25 For the heterologous production of mSOG-loaded encapsulin (Enc-mSOG) in E. coli, the ESigtagged miniSOG (mSOG) cargo was co-expressed with a His-tagged encapsulin (Enc) (Figure 1a). Following their purification by IMAC and SEC, both Enc-mSOG and unloaded Enc underwent biophysical characterization (Figure 1b-e). SDS-PAGE confirmed the co-purification of Enc (Encsubunit; $31.9 \mathrm{kDa}$ ) and mSOG cargo (mSOGESig; $\sim 15.9 \mathrm{kDa}$ ) (Figure 1b, left panel). Densitometric SDS-PAGE gel analysis determined the ratio of Encsubunit to $\mathrm{mSOG}$ cargo and estimated a cargo loading capacity (LC\%) of 7-9\% for Enc-mSOG, which represents $\sim 7 \pm 2$ mSOG molecules packaged per nanocompartment. Under native-PAGE conditions, Enc-mSOG presented high molecular weight bands similar to unloaded Enc, consistent with $T=1$ Enc assemblies (Figure 1b, right panel). The blue-light excitation of mSOG proteins inside Enc-mSOG was detected via fluorescence imaging of the native-PAGE, with no fluorescence observed from empty Enc (Figure 1b, right panel). In order to confirm the correct self-assembly, morphology and size of mSOG-loaded nanocompartments, TEM observations and DLS measurements were performed. TEM images of negatively stained samples showed the accurate formation of Enc-mSOG and empty Enc into spherical nanocompartments (Figure 1c, upper panel). DLS measurement of empty Enc revealed a mean hydrodynamic diameter of $29.0 \pm 2.5 \mathrm{~nm}$ (Figure 1c, lower panel), which was expected to be $\sim 24 \mathrm{~nm}$ based on the crystal structure of Tm encapsulin ( $T=1$; Protein database ID: 3DKT). ${ }^{25}$ This observed enlargement is likely due to the insertion and display of His-tags on the Enc's external surface. This aligns with research by Moon et al, in which Tm encapsulin enlarged to $29.1 \mathrm{~nm}$ after introducing functional peptides into the same 138-139 loop region ${ }^{23}$. Nevertheless, DLS determined that unloaded Enc and Enc-mSOG were both intact and monodisperse 
with mean diameters of $\sim 30 \mathrm{~nm}$, thus mSOG packaging did not significantly alter Enc's morphology or structure.

Next, spectrophotometric analysis was used to gain insight into the effect encapsulation had on the fluorescent properties of $\mathrm{mSOG}$. According to Figure 1d, free mSOG and Enc-mSOG have almost identical fluorescence excitation maxima at 450 $\mathrm{nm}$ (with shoulders at $470 \mathrm{~nm}$ ) and emission maxima at $495 \mathrm{~nm}$ (with shoulders at 525 $\mathrm{nm}$ ). These spectra are consistent with the reported fluorescence spectra of unmodified miniSOG. ${ }^{24}$ However, we observed that mSOG's fluorescence spectra became nosier upon encapsulation and coincided with an $87 \%$ reduction in its fluorescence intensity (Figure 1e). This marked loss of fluorescence could be the result of structural re-arrangements in the FMN-binding region of $\mathrm{mSOG}$ during its encapsulation. Herein, aromatic amino acid residues (i.e. Trp) become exposed to the isoalloxazine ring of the FMN chromophore, leading to $\pi-\pi$ stacking interactions that quench FMN fluorescence, an effect known to occur with other fluorescent flavoproteins. ${ }^{29}$ 
a.

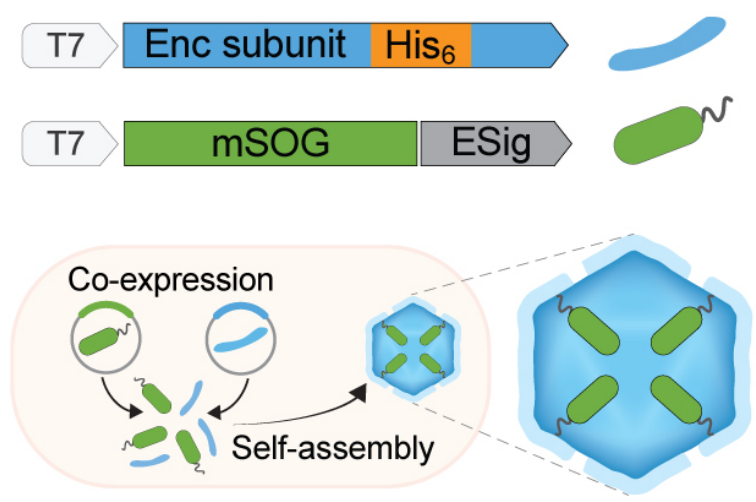

C.
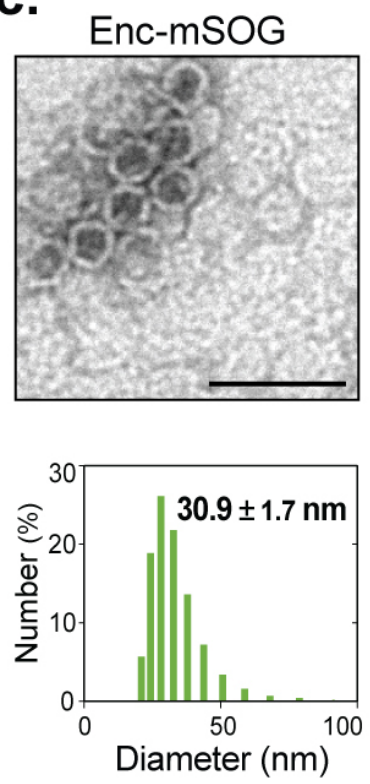

b.
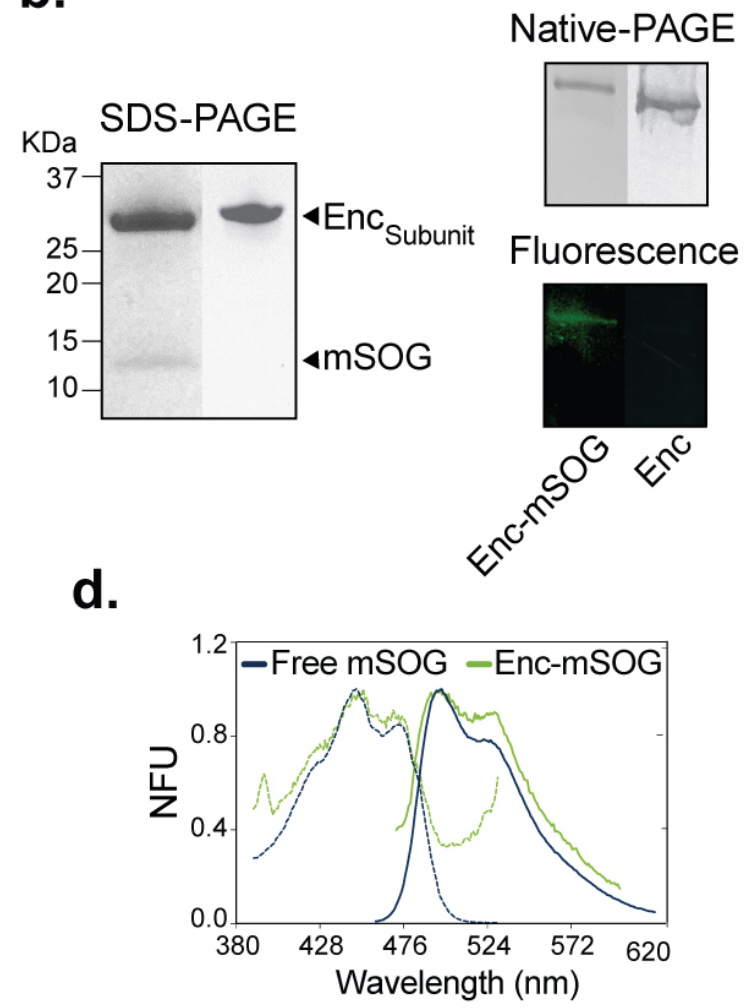

e.

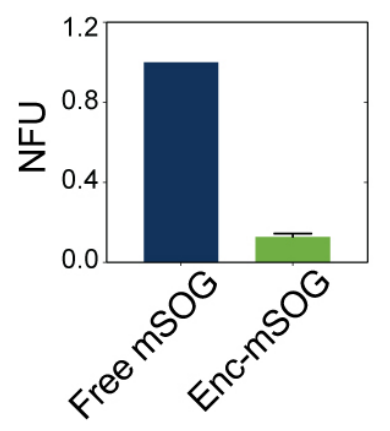

Fig. 1. Design, production and biophysical characterization of mSOG-loaded encapsulin (EncmSOG). (a) (Upper panel) Genetic constructions encoding the Enc subunit from Thermotoga maritima $(\mathrm{Tm})$ (blue) displaying a His-tag (orange) within a surface-exposed loop region, and $\mathrm{mSOG}$ cargo protein (green) C-terminally tagged with an encapsulation signal (ESig, grey). (Lower panel) Heterologous co-expression of encapsulin subunits and ESig-tagged mSOG in E. coli leads to the in vivo self-assembly of cargo-loaded $T m$ Enc $T=1$ nanocompartments. (b) PAGE analysis of Enc-mSOG purified by sequential IMAC and SEC. (Left panel) SDS-PAGE showing the co-purification of the Enc subunit $(31.9 \mathrm{KDa})$ and ESig-tagged $\mathrm{mSOG}$ cargo protein $(14.4 \mathrm{KDa})$. (Right panel) Native-PAGE verifying the self-assembly of Enc-mSOG into cargo-loaded nanocompartments, and in-gel fluorescence of the Native-PAGE confirming encapsulation of fluorescent mSOG cargo. (c) (Upper panel) TEM images of Enc-mSOG and unloaded Enc show their self-assembly into spherical nanocompartments (scale bars $=50 \mathrm{~nm}$ ). (Lower panel) While their respective size distributions measured by DLS indicate average diameters of $\sim 30 \mathrm{~nm}$. (d) Effect of encapsulation on the fluorescence excitation/emission spectra of mSOG. Fluorescence excitation (dashed line) and emission (solid line) spectra of free mSOG and Enc-mSOG given in Normalized Fluorescence Units (NFU). (e) Fluorescence emission intensity $(\mathrm{ex} / \mathrm{em}=485 / 520 \mathrm{~nm})$ of free $\mathrm{mSOG}$ and Enc-mSOG. Each sample contained $500 \mathrm{nM} \mathrm{mSOG}$ equivalent. Error bars represent the mean \pm standard deviation, $n=3$ from three independent experiments. 
"On demand" generation of singlet oxygen from the light-activated Enc-mSOG nanoreactor

Tm encapsulin has multiple 3-4 Å sized surface pores that allow the flow of small metal ions (i.e. $\mathrm{Fe}^{2+/ 3+}$ ) into and out of its internal cavity. ${ }^{3,13}$ As depicted in Figure 2a, we hypothesized that molecular oxygen $\left(\mathrm{O}_{2}\right)$ substrate can diffuse through the open surface pores of Enc-mSOG, enabling its interaction with the mSOG cargo within. EncmSOG can then be activated "on demand" with blue light to photoconvert $\mathrm{O}_{2}$ into ${ }^{1} \mathrm{O}_{2}$. The highly reactive ${ }^{1} \mathrm{O}_{2}$ product subsequently exits Enc-mSOG via its surface pores, allowing it to react with nearby molecules i.e. photosensitization.

To test this hypothesis, the capacity for light-activated Enc-mSOG to generate ${ }^{1} \mathrm{O}_{2}$ in solution was measured using SOSG reagent. SOSG is selectively oxidized by ${ }^{1} \mathrm{O}_{2}$ to emit green fluorescence $(510-550 \mathrm{~nm})$, the intensity of which is relative to the quantity of ${ }^{1} \mathrm{O}_{2}$ produced. Enc-mSOG, free mSOG, and unloaded Enc were mixed with SOSG (in deuterated-HEPES buffer), and each sample irradiated with a blue laser for $10 \mathrm{~min}$ $(450 \mathrm{~nm})$. Afterwards, their fluorescence intensity was measured at $520 \mathrm{~nm}$. Figure 2b shows that unloaded Enc is capable of producing low amounts of ${ }^{1} \mathrm{O}_{2}$ upon laser excitation. Nevertheless, when compared to unloaded Enc, free and encapsulated mSOG generated 2.2-fold and 4.3-fold more ${ }^{1} \mathrm{O}_{2}$, respectively. Unexpectedly, EncmSOG produced 1.9-fold more ${ }^{1} \mathrm{O}_{2}$ generation than free $\mathrm{mSOG}$, likely indicating that Enc has an additive effect when combined with mSOG's own ${ }^{1} \mathrm{O}_{2}$ generation. The relatively low quantities of ${ }^{1} \mathrm{O}_{2}$ produced by unloaded Enc is likely due to the nonspecific absorption of endogenous flavin molecules (e.g. FMN, flavin adenine dinucleotide (FAD), riboflavin) onto the Enc's outer surface, which has been previously observed for $E$. coli produced Tm encapsulin. 30

In order to further characterize the functional effect encapsulation has on $\mathrm{mSOG}^{\prime}{ }^{1} \mathrm{O}_{2}$ generating capacity, the amounts of ${ }^{1} \mathrm{O}_{2}$ produced by free and encapsulated mSOG were compared after different irradiation time periods (0, 5, 10, 15, $20 \mathrm{~min}$ ) (Figure 2c). To account for background ${ }^{1} \mathrm{O}_{2}$ from Enc, we subtracted this value from Enc-mSOG's ${ }^{1} \mathrm{O}_{2}$ generation at each time point. At all tested irradiation times, free and encapsulated mSOG produced similar amounts of ${ }^{1} \mathrm{O}_{2}$ (Figure 2c), indicating that the encapsulation of $\mathrm{mSOG}$ and its subsequent loss of fluorescence intensity (Figure 1e) had no significant adverse effect on its ${ }^{1} \mathrm{O}_{2}$-generating function. However, Enc-mSOG's ${ }^{1} \mathrm{O}_{2}$ production began to plateau at irradiation times longer than $10 \mathrm{~min}$. Notably, without 
the subtraction of Enc's background ${ }^{1} \mathrm{O}_{2}$ production, Enc-mSOG was found to produce statistically greater amounts of ${ }^{1} \mathrm{O}_{2}$ than free mSOG, thus confirming an additive effect when combining Enc and mSOG's ${ }^{1} \mathrm{O}_{2}$ generating capacities (Supplementary Figure 2).

The protein shells of encapsulins are considered robust nanostructures, exhibiting resilience against extreme $\mathrm{pH}$, high temperatures and proteolytic degradation. 1, 23, 25 However, ${ }^{1} \mathrm{O}_{2}$ is highly reactive against some amino acids (e.g. His, Tyr, Met, Cys) and can therefore cause problematic oxidative damage to protein structures. ${ }^{31}$ To assess the physical effect of laser irradiation and ${ }^{1} \mathrm{O}_{2}$ generation on the nanocompartments, we monitored changes to the structure and stability of unloaded Enc and Enc-mSOG after exposure to a blue laser $\left(55 \mathrm{~mW} / \mathrm{cm}^{2}, 10 \mathrm{~min}\right)$. Following the irradiation of empty Enc, DLS measurements indicated a $\sim 31 \%$ increase in its average hydrodynamic diameter from 29.0 to $38.7 \mathrm{~nm}$, while TEM images revealed the presence of predominantly normal spherical nanocompartments with only a small proportion of large amorphous structures (Figure $2 \mathrm{~d}$ and Supplementary Figure 3 ). Thus, laser irradiation alone had a minimal effect on the protein shell's physical properties in accordance with a minimal ${ }^{1} \mathrm{O}_{2}$ generation. In contrast, DLS measurements showed that irradiated Enc-mSOG enlarged $283.5 \%$ from 29.1 to $111.6 \mathrm{~nm}$ (Figure 2e) and lost its monodispersity. Under TEM, a highly heterogeneous population was observed, consisting of enlarged nanocompartments and numerous bulky amorphous structures (Supplementary Figure 3). The loss of Enc-mSOG structural integrity and stability can be attributed to the light-induced activation of its $\mathrm{mSOG}$ cargo, which generates ${ }^{1} \mathrm{O}_{2}$ that could severely damage its surrounding protein shell. ${ }^{32}$ This is consistent with research by Zhen et al., in which ferritin protein nanocages were loaded with the potent chemical photosensitizer $\mathrm{ZnF}_{16} \mathrm{Pc}$, and subsequently destroyed by ${ }^{1} \mathrm{O}_{2}$ generated from the light-activated $\mathrm{ZnF}_{16} \mathrm{Pc}$ cargo. ${ }^{33}$ Furthermore, the ${ }^{1} \mathrm{O}_{2}$-mediated damage to EncmSOG macrostructure could explain why its photoconversion rate begins to plateau after exposure to more than 10 min laser irradiation (Figure 2c). 
a.

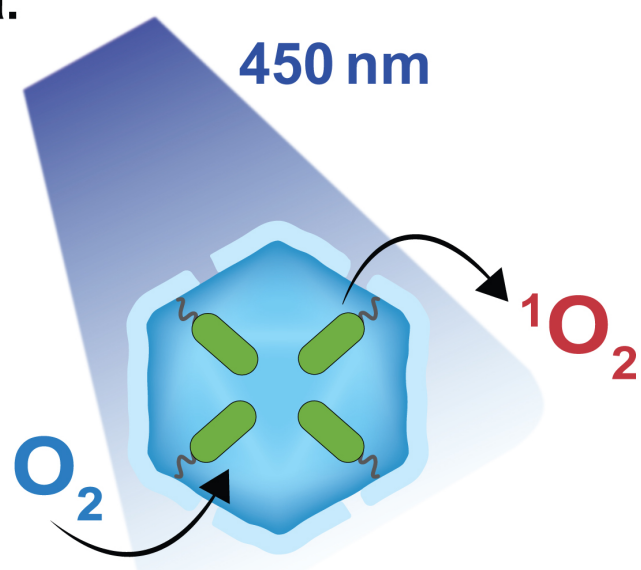

b.

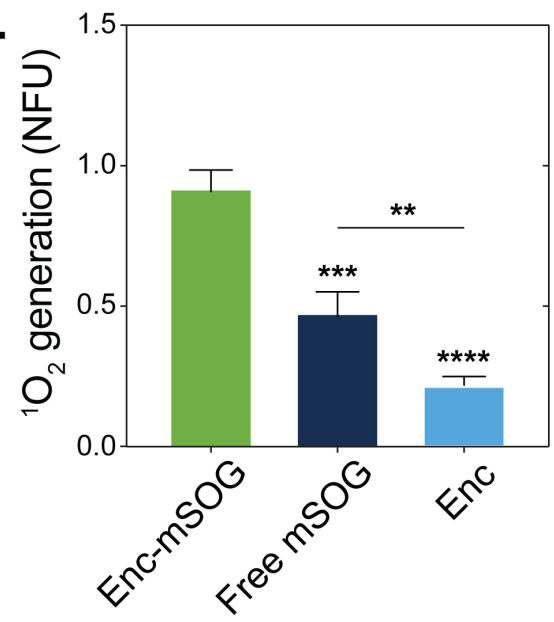

C.

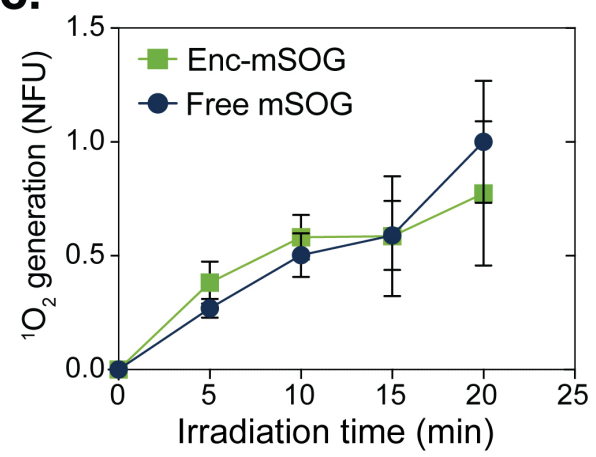

d.

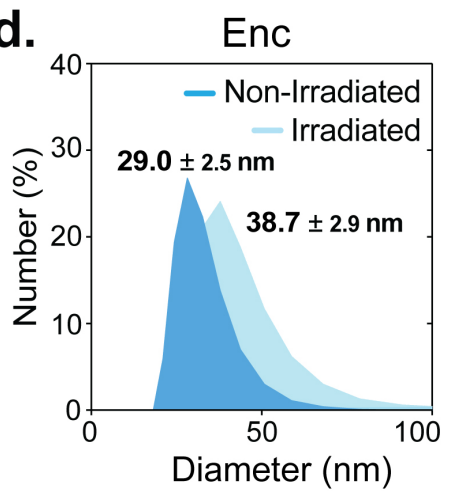

e.

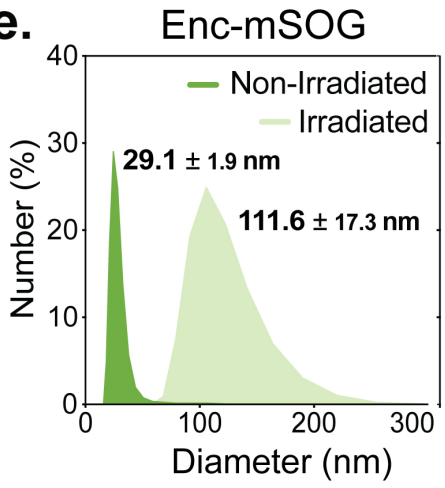

Fig. 2. The "on demand" generation of singlet oxygen by light-activated Enc-mSOG. (a) Illustration showing the production of singlet oxygen $\left({ }^{1} \mathrm{O}_{2}\right)$ by a light-activated Enc-mSOG nanocompartment. In this process, molecular oxygen $\left(\mathrm{O}_{2}\right)$ enters the internal cavity of the nanocompartment via open surface pores where it interacts with encapsulated $\mathrm{mSOG}$ cargo. Upon activation with blue laser light, the mSOG cargo converts $\mathrm{O}_{2}$ (substrate) into ${ }^{1} \mathrm{O}_{2}$ (product), which subsequently diffuses out of the same open surface pores. (b) ${ }^{1} \mathrm{O}_{2}$ generation from Enc-mSOG, free $\mathrm{mSOG}$, and unloaded Enc upon irradiation with a blue laser $(450 \mathrm{~nm})$ at $55 \mathrm{~mW} / \mathrm{cm}^{2}$ for $10 \mathrm{~min} .{ }^{1} \mathrm{O}_{2}$ production was determined by the fluorescence intensity of oxidized SOSG, (NFU). Error bars represent the mean \pm standard deviation, one-way ANOVA, Tukey, $n=3\left({ }^{*} p \leq 0.05,{ }^{* *} p \leq 0.01,{ }^{* * *} p \leq 0.001,{ }^{* * * *} p \leq 0.0001\right)$. (c) ${ }^{1} \mathrm{O}_{2}$ production by free and encapsulated $\mathrm{mSOG}$ after laser irradiation at $450 \mathrm{~nm}\left(55 \mathrm{~mW} / \mathrm{cm}^{2}\right)$ for $0,5,10,15$ and $20 \mathrm{~min} .{ }^{1} \mathrm{O}_{2}$ generation was measured using SOSG. It should be noted that the background ${ }^{1} \mathrm{O}_{2}$ produced by unloaded Enc was subtracted from the total ${ }^{1} \mathrm{O}_{2}$ produced by Enc-mSOG. Error bars represent the mean \pm standard deviation $n=3$. (d) and (e) DLS-measured size distributions of unloaded Enc and Enc-mSOG before (Non-Irradiated) and after (Irradiated) laser excitation at $450 \mathrm{~nm}\left(55 \mathrm{~mW} / \mathrm{cm}^{2}\right)$ for $10 \mathrm{~min}$.

\section{Evaluating the photosensitizing function of Enc-mSOG in an in vitro model of} photodynamic therapy (PDT)

In vitro studies have demonstrated miniSOG's potential as a biological photosensitizer for PDT. ${ }^{34,35}$ To eliminate tumour cells, PDT relies on light-induced photosensitizers that convert intracellular oxygen into ROS, which damage cellular componentry and cause cell death. ${ }^{36}$ As a proof-of-concept (Figure 3a), we decided to explore whether 
ROS produced by the light-activated Enc-mSOG nanoreactor was sufficient to trigger cellular photodynamic responses in an in vitro model of human lung cancer.

First, the cytotoxicity of unloaded Enc, free mSOG and Enc-mSOG was assessed in dark conditions. Previous work by Deyev demonstrated that miniSOG tagged with a cancer-specific antibody mediated targeted PDT in vitro, exerting its maximal phototoxic effect against cancer cells at a concentration of 500 nM. ${ }^{34}$ Accordingly, A549 human lung cancer cells were pre-incubated with $4.8 \mathrm{mM}$ of unloaded Enc and $500 \mathrm{nM}$ mSOG or Enc-mSOG for increasing periods of time (2, 4, 8 and $12 \mathrm{~h}$ ) in the dark, after which viability was measured by MTT assay. In the absence of light activation, free mSOG and Enc-mSOG showed no significant cytotoxicity when compared to untreated cells (Figure $3 b$ ). This was also the found to be the case for unloaded Enc (Supplementary Figure 5a).

Next, we sought to evaluate the cytotoxicity of unloaded Enc and free and encapsulated mSOG in conjunction with blue-light irradiation i.e. phototoxicity. Initially, the effect of laser irradiation on live cells was assessed by exposing A549 cells to a blue laser $\left(55 \mathrm{~mW} / \mathrm{cm}^{2}\right)$. An irradiation time of up to $10 \mathrm{~min}$ had no significant effect on cell viability (Supplementary Figure 4) and was therefore used to study in vitro phototoxicity. Herein, A549 cells were treated using the same conditions outlined above, followed by 10 min blue-light irradiation, with cell viability then determined via MTT assay. As shown in Figure 3c, A549 cells showed no significant changes in viability after pre-incubation with free $\operatorname{mSOG}(2,4,8$ and $12 \mathrm{~h})$ and blue-light irradiation. This is consistent with reports in which unmodified free miniSOG was unable to efficiently bind or enter cancer cells and exert its phototoxic effect ${ }^{34}$. Likewise, unloaded Enc elicited no significant decrease in cell viability upon light irradiation (Supplementary Figure $5 b$ ), suggesting that ${ }^{1} \mathrm{O}_{2}$ generated by Enc alone is too low to be cytotoxic. In contrast, cells pre-incubated with Enc-mSOG (2, 4, 8 and $12 \mathrm{~h}$ ) and exposed to laser irradiation exhibited a decrease in viability for each treatment tested. For instance, the viability of cells pre-incubated with Enc-mSOG for 8 or $12 \mathrm{~h}$ were significantly reduced by $\sim 34 \%$. Thus, Enc serves as a viable nanocarrier for $\mathrm{mSOG}$, localizing it in tumour cell membranes and interiors, where it can be triggered by light to induce oxidative damage that lowers cell viability. 
a.

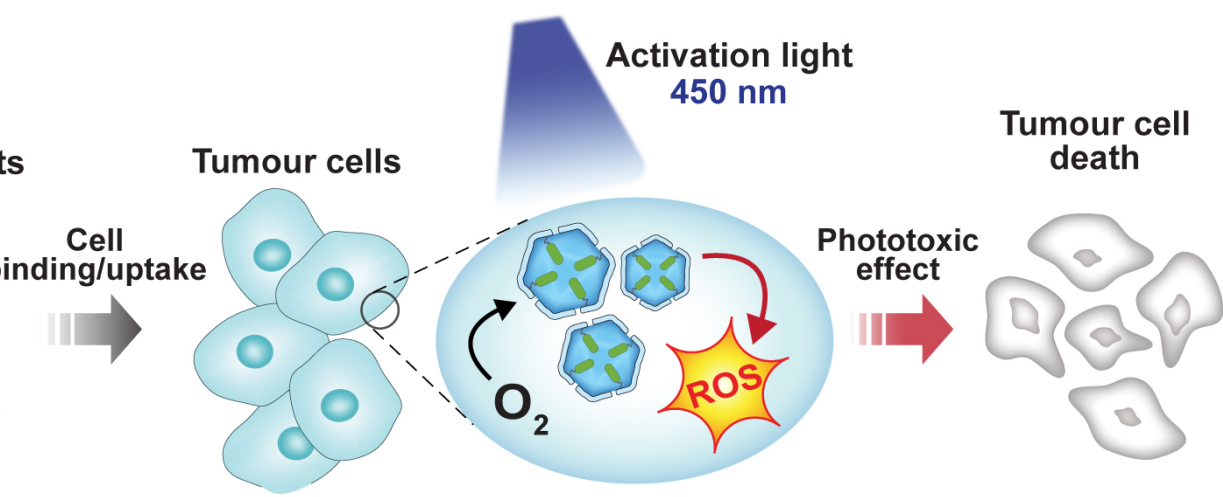

b.

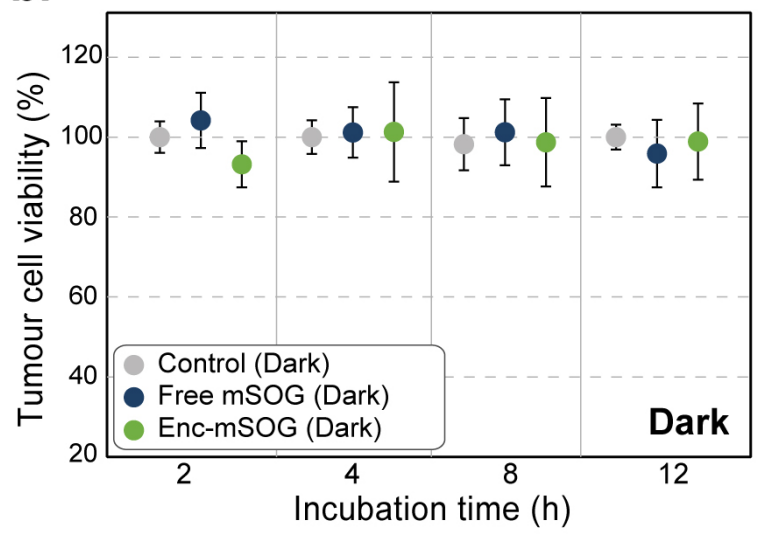

c.

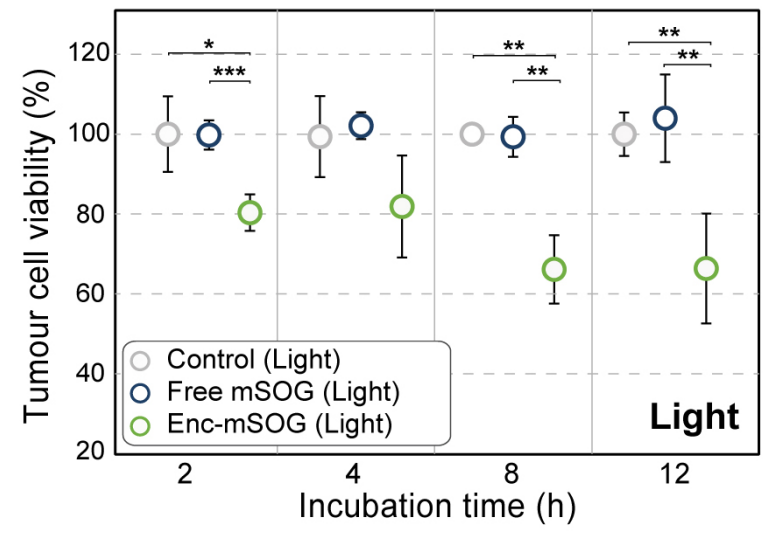

Figure 3. Testing the capacity of light-activated Enc-mSOG to induce photodynamic responses in an in vitro cancer model. (a) Schematic diagram showing the proposed delivery, activation and phototoxic effect of Enc-mSOG nanocompartments. Photosensitizing Enc-mSOG enters tumour cells via endocytosis. Upon photoexcitation with blue light, Enc-mSOG converts intracellular $\mathrm{O}_{2}$ into cytotoxic ROS (e.g. ${ }^{1} \mathrm{O}_{2}$ ) that induces tumour cell death. (b) Cytotoxicity of free and encapsulated mSOG: viability of A549 cells after incubation without (control) or with mSOG or Enc-mSOG for different times (2, 4, 8, and $12 \mathrm{~h}$ ) in the dark. Cell viability was subsequently determined by MTT assay. Error bars represent the mean \pm standard deviation $(p \leq 0.05)$, one-way ANOVA, Dunnett $T 3, n=6$ from two independent experiments. (c) Phototoxicity of free and encapsulated mSOG: Viability of A549 cells incubated without (control) or with free $\mathrm{mSOG}$ or Enc-mSOG for different times (2, 4, 8, and $12 \mathrm{~h}$ ) in the dark, followed by blue laser irradiation at $450 \mathrm{~nm}\left(55 \mathrm{~mW} / \mathrm{cm}^{2}\right)$ for $10 \mathrm{~min}$. Cell viability was quantified via MTT assay. Error bars represent the mean \pm standard deviation $\left({ }^{*} p \leq 0.05,{ }^{* *} p \leq 0.01\right)$, one-way ANOVA, Dunnett T3, $\mathrm{n}=6$ from two independent experiments. 


\section{Conclusion}

In summary, we reversed the oxidative stress response functions of $\mathrm{Tm}$ encapsulin, programming it to instead serve as a light-responsive encapsulin nanoreactor that generates ROS "on demand" and triggers photosensitization reactions. To realise this objective, the protein photosensitizer miniSOG was tagged with the Tm ESig and selectively packaged into $\mathrm{Tm}$ encapsulin, resulting in a self-assembled Enc-mSOG nanoreactor that showed similar structural properties to unloaded nanocompartments.

Upon encapsulation, mSOG retained its fluorescence spectra but lost its fluorescence intensity, potentially due to structural rearrangements. Importantly, we found that confinement had no effect on mSOG's ability to photoconvert $\mathrm{O}_{2}$ to ${ }^{1} \mathrm{O}_{2}$ under bluelight excitation. Specifically, free and encapsulated mSOG generated comparable amounts of ${ }^{1} \mathrm{O}_{2}$, and empty Enc produced small quantities of ${ }^{1} \mathrm{O}_{2}$. Enc-mSOG's total ${ }^{1} \mathrm{O}_{2}$ production confirmed an additive effect between mSOG cargo and Enc, a positive attribute that provides a unique advantage over the use of free mSOG alone. Additionally, these findings infer the adsorption of endogenous photosensitizing flavins (e.g. FMN and FAD) onto Tm encapsulin, offering insight into its physiological function.

After prolonged blue-light exposure, Enc-mSOG began to show signs of structural deterioration, which coincided with a slow plateau in its photoconversion rate. These adverse effects were likely caused by the rapid generation of destructive ${ }^{1} \mathrm{O}_{2}$ inside Enc-mSOG's protein shell. ${ }^{31}$ Given their structural adaptability, encapsulin protein shells could be re-engineered to become more resilient towards ROS. For instance, residues prone to ${ }^{1} \mathrm{O}_{2}$ oxidation could be substituted with ${ }^{1} \mathrm{O}_{2}$-insensitive residues, while surface pores could be enlarged to avoid product accumulation and increase substrate turnover. ${ }^{37,} 38$

As a proof of concept, light-activated Enc-mSOG triggered photosensitized oxidation reactions that significantly lowered the viability of lung cancer cells. To our knowledge, this is the first time encapsulins have been used to deliver protein cargo in a therapeutic manner. Nevertheless, higher intracellular doses of Enc-mSOG will be required to maximize its efficacy in future PDT studies. This could be accomplished by engineering Enc-mSOG's external surface to display targeting ligands (e.g. peptides and antibodies) that increase cancer cell uptake. In an example of this approach, Tm 
encapsulin was modified to co-display hepatocellular carcinoma (HCC)-targeting peptides and anticancer drugs, resulting in the effective delivery of drugs into HCC cells. ${ }^{23}$

Due to the high spatial and temporal resolution offered by light, we expect that the novel Enc-mSOG nanoreactor can be selectively activated to initiate and/or modulate other ROS-sensitive processes with technological, biological and therapeutic relevance. Ultimately, this work illustrates the remarkable versatility of encapsulin nanoreactors, paving the way towards the establishment of new techniques and mechanisms that can more precisely control their activities. 


\section{References}

1. Lau, Y. H.; Giessen, T. W.; Altenburg, W. J.; Silver, P. A., Prokaryotic nanocompartments form synthetic organelles in a eukaryote. Nature Communications 2018, $9(1), 1311$.

2. $\quad$ Sigmund, F.; Massner, C.; Erdmann, P.; Stelzl, A.; Rolbieski, H.; Desai, M.; Bricault, S.; Worner, T. P.; Snijder, J.; Geerlof, A.; Fuchs, H.; Hrabe de Angelis, M.; Heck, A. J. R.; Jasanoff, A.; Ntziachristos, V.; Plitzko, J.; Westmeyer, G. G., Bacterial encapsulins as orthogonal compartments for mammalian cell engineering. Nat Commun 2018, 9 (1), 1990.

3. $\quad$ Sigmund, F.; Pettinger, S.; Kube, M.; Schneider, F.; Schifferer, M.; Schneider, S.; Efremova, M. V.; Pujol-Martí, J.; Aichler, M.; Walch, A.; Misgeld, T.; Dietz, H.; Westmeyer, G. G., Iron-Sequestering Nanocompartments as Multiplexed Electron Microscopy Gene Reporters. ACS Nano 2019, 13 (7), 8114-8123.

4. Rother, M.; Nussbaumer, M. G.; Renggli, K.; Bruns, N., Protein cages and synthetic polymers: a fruitful symbiosis for drug delivery applications, bionanotechnology and materials science. Chem Soc Rev 2016, 45 (22), 6213-6249.

5. Mosayebi, M.; Shoemark, D. K.; Fletcher, J. M.; Sessions, R. B.; Linden, N.; Woolfson, D. N.; Liverpool, T. B., Beyond icosahedral symmetry in packings of proteins in spherical shells. Proceedings of the National Academy of Sciences 2017, 114 (34), 9014-9019. 6. Chen, A. H.; Silver, P. A., Designing biological compartmentalization. Trends in Cell Biology 2012, 22 (12), 662-670.

7. Sutter, M.; Boehringer, D.; Gutmann, S.; Gunther, S.; Prangishvili, D.; Loessner, M. J.; Stetter, K. O.; Weber-Ban, E.; Ban, N., Structural basis of enzyme encapsulation into a bacterial nanocompartment. Nat Struct Mol Biol 2008, 15 (9), 939-47.

8. McHugh, C. A.; Fontana, J.; Nemecek, D.; Cheng, N.; Aksyuk, A. A.; Heymann, J. B.; Winkler, D. C.; Lam, A. S.; Wall, J. S.; Steven, A. C.; Hoiczyk, E., A virus capsid-like nanocompartment that stores iron and protects bacteria from oxidative stress. Embo j 2014, 33 (17), 1896-911.

9. Giessen, T. W.; Orlando, B. J.; Verdegaal, A. A.; Chambers, M. G.; Gardener, J.; Bell, D. C.; Birrane, G.; Liao, M.; Silver, P. A., Large protein organelles form a new iron sequestration system with high storage capacity. eLife 2019, 8, e46070.

10. Contreras, H.; Joens, M. S.; McMath, L. M.; Le, V. P.; Tullius, M. V.; Kimmey, J. M.; Bionghi, N.; Horwitz, M. A.; Fitzpatrick, J. A.; Goulding, C. W., Characterization of a Mycobacterium tuberculosis nanocompartment and its potential cargo proteins. $J$ Biol Chem 2014, 289 (26), 18279-89.

11. He, D.; Hughes, S.; Vanden-Hehir, S.; Georgiev, A.; Altenbach, K.; Tarrant, E.; Mackay, C. L.; Waldron, K. J.; Clarke, D. J.; Marles-Wright, J., Structural characterization of encapsulated ferritin provides insight into iron storage in bacterial nanocompartments. eLife 2016, 5 .

12. Giessen, T. W.; Silver, P. A., Widespread distribution of encapsulin nanocompartments reveals functional diversity. Nature microbiology 2017, 2, 17029.

13. Nichols, R. J.; Cassidy-Amstutz, C.; Chaijarasphong, T.; Savage, D. F., Encapsulins: molecular biology of the shell. Critical Reviews in Biochemistry and Molecular Biology 2017, 52 (5), 583-594.

14. Barnett, M. E.; Baran, T. M.; Foster, T. H.; Wojtovich, A. P., Quantification of lightinduced miniSOG superoxide production using the selective marker, 2-hydroxyethidium.

Free Radic Biol Med 2018, 116, 134-140. 
15. Ogilby, P. R., Singlet oxygen: there is indeed something new under the sun. Chem Soc Rev 2010, 39 (8), 3181-209.

16. Chen, X.; Winters, C.; Crocker, V.; Lazarou, M.; Sousa, A. A.; Leapman, R. D.; Reese, T. S., Identification of PSD-95 in the Postsynaptic Density Using MiniSOG and EM Tomography. Front Neuroanat 2018, 12, 107-107.

17. Alonso-de Castro, S.; Cortajarena, A. L.; López-Gallego, F.; Salassa, L., Bioorthogonal Catalytic Activation of Platinum and Ruthenium Anticancer Complexes by FAD and Flavoproteins. Angewandte Chemie 2018, 130 (12), 3197-3201.

18. Jiang, H. N.; Li, Y.; Jiang, W. Y.; Cui, Z. J., Cholecystokinin 1 Receptor - A Unique G Protein-Coupled Receptor Activated by Singlet Oxygen (GPCR-ABSO). Front Physiol 2018, 9, 497.

19. Burgers, P. P.; Ma, Y.; Margarucci, L.; Mackey, M.; van der Heyden, M. A.; Ellisman, M.; Scholten, A.; Taylor, S. S.; Heck, A. J., A small novel A-kinase anchoring protein (AKAP) that localizes specifically protein kinase A-regulatory subunit I (PKA-RI) to the plasma membrane. J Biol Chem 2012, 287 (52), 43789-97.

20. Lin, J. Y.; Sann, S. B.; Zhou, K.; Nabavi, S.; Proulx, C. D.; Malinow, R.; Jin, Y.; Tsien, R. Y., Optogenetic inhibition of synaptic release with chromophore-assisted light inactivation (CALI). Neuron 2013, 79 (2), 241-253.

21. Makhijani, K.; To, T. L.; Ruiz-Gonzalez, R.; Lafaye, C.; Royant, A.; Shu, X., Precision Optogenetic Tool for Selective Single- and Multiple-Cell Ablation in a Live Animal Model System. Cell chemical biology 2017, 24 (1), 110-119.

22. Proshkina, G. M.; Shramova, E. I.; Shilova, O. N.; Ryabova, A. V.; Deyev, S. M., Phototoxicity of flavoprotein miniSOG induced by bioluminescence resonance energy transfer in genetically encoded system NanoLuc-miniSOG is comparable with its LED-excited phototoxicity. J Photochem Photobiol B 2018, 188, 107-115.

23. Moon, H.; Lee, J.; Min, J.; Kang, S., Developing genetically engineered encapsulin protein cage nanoparticles as a targeted delivery nanoplatform. Biomacromolecules 2014, 15 (10), 3794-801.

24. Shu, X.; Lev-Ram, V.; Deerinck, T. J.; Qi, Y.; Ramko, E. B.; Davidson, M. W.; Jin, Y.; Ellisman, M. H.; Tsien, R. Y., A genetically encoded tag for correlated light and electron microscopy of intact cells, tissues, and organisms. PLoS biology 2011, 9 (4), e1001041. 25. Cassidy-Amstutz, C.; Oltrogge, L.; Going, C. C.; Lee, A.; Teng, P.; Quintanilla, D.; East-Seletsky, A.; Williams, E. R.; Savage, D. F., Identification of a Minimal Peptide Tag for in Vivo and in Vitro Loading of Encapsulin. Biochemistry 2016, 55 (24), 3461-8.

26. Lawrence, A.-M.; Besir, H. U. S., Staining of proteins in gels with Coomassie G-250 without organic solvent and acetic acid. Journal of visualized experiments : JoVE 2009, (30), 1350.

27. Schneider, C. A.; Rasband, W. S.; Eliceiri, K. W., NIH Image to ImageJ: 25 years of image analysis. Nat Methods 2012, 9 (7), 671-5.

28. Iselt, M.; Holtei, W.; Hilgard, P., The tetrazolium dye assay for rapid in vitro assessment of cytotoxicity. Arzneimittel-Forschung 1989, 39 (7), 747-9.

29. Mukherjee, A.; Weyant, K. B.; Walker, J.; Schroeder, C. M., Directed evolution of bright mutants of an oxygen-independent flavin-binding fluorescent protein from Pseudomonas putida. Journal of Biological Engineering 2012, 6 (1), 20.

30. Caleb, C.-A. Shellular biology - exploring the biochemistry and physiology of a protein nanocompartment. Disertation, University of California, Berkeley, 2017.

31. Gracanin, M.; Hawkins, C. L.; Pattison, D. I.; Davies, M. J., Singlet-oxygen-mediated amino acid and protein oxidation: formation of tryptophan peroxides and decomposition products. Free Radic Biol Med 2009, 47 (1), 92-102. 
32. Michaeli, A.; Feitelson, J., Reactivity of singlet oxygen toward proteins: the effect of structure in basic pancreatic trypsin inhibitor and in ribonuclease A. Photochem Photobiol 1997, 65 (2), 309-15.

33. Zhen, Z.; Tang, W.; Guo, C.; Chen, H.; Lin, X.; Liu, G.; Fei, B.; Chen, X.; Xu, B.; Xie, J., Ferritin nanocages to encapsulate and deliver photosensitizers for efficient photodynamic therapy against cancer. ACS Nano 2013, 7 (8), 6988-96.

34. Mironova, K. E.; Proshkina, G. M.; Ryabova, A. V.; Stremovskiy, O. A.; Lukyanov, S. A.; Petrov, R. V.; Deyev, S. M., Genetically encoded immunophotosensitizer 4D5scFvminiSOG is a highly selective agent for targeted photokilling of tumor cells in vitro. Theranostics 2013, 3 (11), 831-40.

35. Proshkina, G. M.; Shilova, O. N.; Ryabova, A. V.; Stremovskiy, O. A.; Deyev, S. M., A new anticancer toxin based on HER2/neu-specific DARPin and photoactive flavoprotein miniSOG. Biochimie 2015, 118, 116-122.

36. Serebrovskaya, E. O.; Edelweiss, E. F.; Stremovskiy, O. A.; Lukyanov, K. A.; Chudakov, D. M.; Deyev, S. M., Targeting cancer cells by using an antireceptor antibodyphotosensitizer fusion protein. Proceedings of the National Academy of Sciences 2009, 106 (23), 9221-9225.

37. Dogra, V.; Li, M.; Singh, S.; Li, M.; Kim, C., Oxidative post-translational modification of EXECUTER1 is required for singlet oxygen sensing in plastids. Nature Communications 2019, 10 (1), 2834.

38. Williams, E. M.; Jung, S. M.; Coffman, J. L.; Lutz, S., Pore Engineering for Enhanced Mass Transport in Encapsulin Nanocompartments. ACS Synthetic Biology 2018, 7 (11), 25142517.

\section{Acknowledgments}

D.D. is supported by an international Macquarie University Research Excellence Scholarship (iMQRES), Sydney Vital Research Scholar Award, and the Commonwealth Scientific and Industrial Research Organisation (CSIRO) PhD Scholarship Program in Synthetic Biology. A.C. is supported by a Cancer Institute New South Wales Early Career Fellowship (Project Number: ECF171114), the Cancer Australia Priority-driven Collaborative Cancer Research Scheme (Project Number: 1182082), and the Australian Research Council (CE140100003).

\section{Author contributions}

D.D co-designed the research, generated all nanocompartment constructs, conducted all physical and functional characterization work, performed data analysis and wrote the manuscript. X.V. constructed the laser set-up, supported laser irradiation experiments and revised the manuscript. A.S. supervised the project and revised the manuscript. A.C. conceptualized and co-designed the study, supervised the project and wrote the manuscript. 
bioRxiv preprint doi: https://doi.org/10.1101/2020.06.06.138305; this version posted June 7, 2020. The copyright holder for this preprint (which was not certified by peer review) is the author/funder. All rights reserved. No reuse allowed without permission.

\section{Additional information}

Competing interests: The authors declare no competing interests. 